\title{
Determination of chromatic aberration in the human eye by means of Fresnel propagation theory
}

\author{
David Mas, Jorge Perez, Carlos Illueca, Julian Espinosa, Consuelo Hernandez, Carmen Vazquez, \\ and Juan J. Miret
}

Dept. of Optics, University of Alicante. P.O. Box 99. 03080 Alicante SPAIN

\begin{abstract}
In this communication, the authors have determined the longitudinal chromatic aberrations in real eyes. The method that has been used combines real data of corneal morphology, central thickness of crystalline lens and biometric measures of axial length together with numerical calculation of the propagation process. The curvature of the crystalline lens has been adjusted to different curvature models and refractive index distributions. The wavelength dependence of all ocular media has been modelled through the Cauchy formula. Propagation through anterior and posterior chambers has been accomplished through numerical calculation of diffraction integral instead of classical ray-tracing approach This imposes serous restrictions on the number of samples that are needed for a full propagation process. If we are only interested in amplitude calculations the method consists of evaluating propagation from cornea to crystalline lens with a spectrum propagation method. Propagation from the lens to the best image plane is accomplished by a direct calculation of Fresnel integral. With this model, we have obtained the refraction chromatic difference in diopters for several eyes. Results are compared with real measures of the chromatic aberration, showing a good agreement with numerical calculations. The capabilities of the technique have been demonstrated by applying the method to the study of the chromatic aberration of a keratoconus.
\end{abstract}

Keywords: Physiological Optics, Beam Propagation, Chromatic Aberrations

\section{INTRODUCTION}

Aberrations of the human eye have been intensely studied in the last decades. Both principal contributors to the aberrations in the human eye are the cornea and the crystalline lens, and their influence on the longitudinal chromatic aberration (LCA) and the transversal chromatic aberration (TCA) have been measured by several authors ${ }^{1-9}$.

Historically, LCA have been modelled using water-eye models. Chromatic aberration of the reduced eye is attributed solely to the variation of the refractive index of water with wavelength ${ }^{10}$. In a real eye, the corneal and crystalline lens profiles affect to the chromatic aberration. Published measurements of the magnitude of chromatic aberration have shown that this simplified model does not fit the data well for short wavelengths.

Unfortunately, a model that takes into account the different surfaces in the eye has a large amount of spherical aberration so the best image plane can not be exactly calculated. Thus, aberrations are measured using psychophysical methods of best focus determination. Thibos et al. ${ }^{6}$ measured experimentally the chromatic variation of refractive error in human eyes by the two colours Vernier method. Although this method provides psychophysical measurements it has less measurement error, better acuity ${ }^{11}$ and it is less vulnerable to criterion differences than a method based on best focus determination. Longitudinal chromatic aberration has also been measured objectively by Charman and Jennings ${ }^{3}$ and Rynders, et al. ${ }^{7}$, who used retinoscopic methods. Fernandez et al. ${ }^{12}$ measured the ocular aberrations in the near infrared part of the spectrum with a Hartmann-Shack wave-front sensor.

The method here proposed is independent of psychophysical measurements and avoids criterion differences. Moreover, previous methods were limited to use a reduced number of wavelengths while we can use all the wavelengths we think are necessaries. 
Numerical analysis and simulation of image formation in the eye are important tools to understand the optical performance of the human eye. Wave optics applied to the problem drives to complex but robust algorithms. Among all the available algorithms we have centred our attention on Fourier-based Fresnel propagation algorithms ${ }^{13,14}$.

Combination of real data of comea and axial lengths of the eye together with a crystalline model and Fresnel propagation algorithms allows realistic simulations of light propagation inside the eye $\mathrm{e}^{15-17}$. The procedure consists of taking clinical data from real subjects. We take corneal topography and we measure the thickness of the crystalline lens, the real anterior chamber depth and the total axial length of the eye. With these data, we calculate light patterns at different distances inside the eye and perform a complete analysis of the image formation process in real time ${ }^{18}$. We have used this method to analyze chromatic aberration of the human eye and to describe optical quality in different subjects. We also present our conclusions compared with experimental results of the bibliography.

\section{METHOD}

First, we take topographic data with a Zeiss Humphrey ATLAS ${ }^{\circ}$ topographer and reconstruct corneal morphology ${ }^{16}$. We have discarded the maps that present no data areas within a zone of radius of $3.6 \mathrm{~mm}$ from the centre of the topography. With these maps, the cornea surface is modelled as a phase element.

Since distances in the anterior chamber are very short, we apply an angular spectrum-based algorithm to describe light propagation from the cornea to the immediately previous plane of the crystalline lens ${ }^{19}$. Let us consider a plane wave that enters the cornea. The light distribution at the entrance plane of the crystalline lens can be numerically evaluated as:

$$
u_{\mu}=D F T^{-1}\left\{\exp \left(i \frac{2 \pi n_{a}(\lambda)}{\lambda}\left(z_{A C}-z_{r e f}\right) \sqrt{1-\left(\frac{\lambda}{n_{a}(\lambda)}\right)^{2} \frac{\tilde{m}^{2}}{\Delta x_{0}^{2}}}\right) D F T\left[\exp \left\{i \frac{2 \pi}{\lambda}\left[z\left(\frac{m \Delta x_{0}}{N}\right)+n_{a}(\lambda)\left(z_{r e f}-z\left(\frac{m \Delta x_{0}}{N}\right)\right)\right]\right\}\right]\right\}
$$

where $m, \tilde{m}$ and $\mu$ are discrete indices in space, Fourier and Fresnel domain, respectively. $z_{r e f}$ is the distance between the apex of the cornea and the exit plane of this element, $z_{A C}$ is the distance between the apex of the cornea and the first surface of the lens, $N$ is the number of samples, $D F T$ is the acronym of discrete Fourier transform. $\Delta x_{0}$ is the size of the object support window and the variable $x$ has been discretized as $x=m \Delta x_{0} / N$. Finally $n_{a}(\lambda)$ is the index of the aqueous humour (see fig. 1).

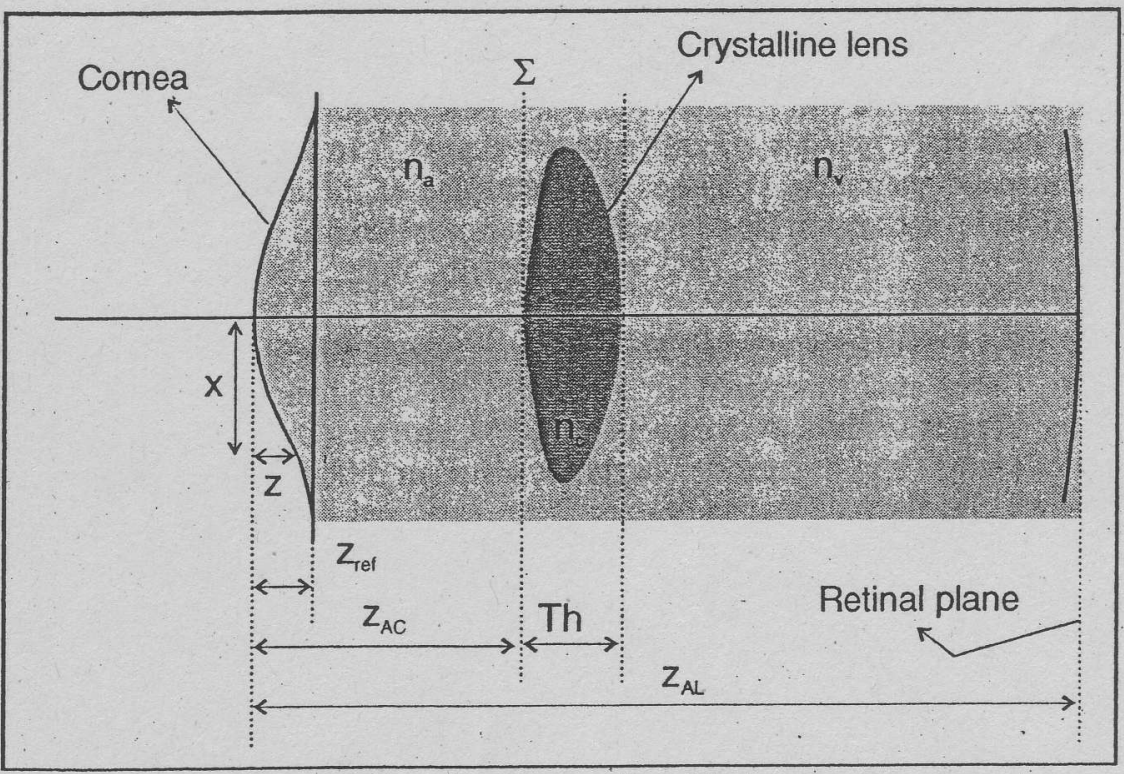

Figure 1: Schematic representation of the human eye. 
The variation with the wavelength that has been used here for all refractive indexes inside the eye has been done according to Cauchy's approximate equation ${ }^{20}$.

$$
n(\lambda) \simeq A+B / \dot{\lambda}^{2}+C / \lambda^{4}+D / \lambda^{6}
$$

Correct sampling of the above written expression needs fulfilling Nyquist criterion. This provides a limitation in the number of samples given by:

$$
N \geq \frac{\Delta x_{0}^{2} n_{a}(\lambda)}{\lambda z_{c}}
$$

where $z_{c}$ is the paraxial focal length of the convergent element under consideration ${ }^{14}$.

Let us take, for example a cornea with a typical paraxial focal length of $z_{c}=32 \mathrm{~mm}$. For an entrance pupil of $4 \mathrm{~mm}$, we select an input window of $\Delta x_{0}=5 \mathrm{~mm}$. We will analyze the spectrum included between $400 \mathrm{~nm}$ and $900 \mathrm{~nm}$. Note that $N$ explicitly depends of $\lambda$, so the necessary number of samples will change between 2609 and 1160 . We take $N=2609$ that corresponds to $\lambda=400 \mathrm{~nm}$ insuring a sufficient number of samples for other wavelengths.

Come to this point, the crystalline lens must be incorporated as an element that affects the phase of the propagated wave. This element has been modelled mathematically as a conicoid described by the equation

$$
x^{2}+y^{2}+(1+Q) z^{2}-2 R z=0
$$

where $R$ and $Q$ are the radius of curvature and the asphericity parameter respectively and both depend on the age, and $x$, $y$, and $z$ are spatial coordinates, the $z$ axis being the optical axis.

The parameters used in the study are those estimated by Dubbelman et.${ }^{21}{ }^{21}$.

$$
\begin{aligned}
& Q_{\text {ant }}=-6.4+0.03 \times \text { age } \\
& Q_{\text {post }}=-6+0.07 \times a g e \\
& R_{\text {ant }}=12.9-0.057 \times \text { age } \\
& R_{\text {post }}=-6.2+0.012 \times \text { age }
\end{aligned}
$$

where age is the age of the subject in years.

Aside of the morphology of the crystalline lens, its refractive index distribution is a critical parameter. Siedlecki et al. ${ }^{22}$ propose a radial crystalline lens. model in which the refractive index changes only with the distance to the optical axis. The authors of the model have compared it with the models of Emsley ${ }^{23}$, Gullstrand No. 1 and No. $2^{24}$, Le Grand and $\mathrm{Hage}^{25}$, and Kooijman ${ }^{26}$, showing that the proposed model provides a better retinal image quality and lower spherical aberration than all the other eye models tested. The variation of the index of refraction of the above mentioned model can be expressed by

$$
n(X, \lambda)=n_{1}(\lambda)-\left(n_{1}(\lambda)-n_{2}(\lambda)\right)\left[\frac{1-\exp (-\beta X)}{1-\exp \left(-\beta X_{\max }\right)}\right]
$$

where $n_{1}(\lambda)$ and $n_{2}(\lambda)$ are the indexes of the lens core and periphery respectively, $\beta$ is the exponential coefficient, $X$ is the distance from the optical axis and $X_{\max }$ is the equatorial radius of the lens. The exponential coefficient $\beta$ has been fixed by Siedlecki to $\beta=-0.91 \mathrm{~mm}^{-1}$.

With all these parameters we can calculate the optical path covered by the light when passing through crystalline lens. Again, considering the lens as a phase element, we will determine the light distribution at the exit plane. Now, a second 
propagation takes place between the second surface of the lens and an output desired plane. For the calculation of a light pattern in this plane, we use a propagation algorithm based on a direct method of determination of the Fresnel integral ${ }^{14}$. Method determines the diffracted light field correctly around the distance of convergence. The light distribution at the output plane at a distance $z$ :

$$
u_{\tilde{m}}(z, \lambda)=\exp \left(i \pi \frac{\lambda\left(z-T h-z_{A C}\right)}{n_{v}(\lambda) \Delta x_{0}^{2}} \tilde{m}^{2}\right) D F T\left[u_{\Sigma}\left(\frac{m \Delta x_{0}}{N}\right) \exp \left(i \frac{2 \pi n_{a}(\lambda)}{\lambda} O p c\left(\lambda, \frac{m \Delta x_{0}}{N}\right)\right) \exp \left(i \pi \frac{n_{v}(\lambda) \Delta x_{0}^{2}}{\lambda\left(z-T h-z_{A C}\right) N^{2}} m^{2}\right)\right]
$$

where $O p c$ is the optical path length in the crystalline lens and $T h$ is the lens thickness, which has been measured with ultrasonography.

Once we obtain the output light pattern, we need to determine the best image position to obtain the chromatic difference of refraction in that plane. Recent studies ${ }^{17,27-29}$ suggest that image quality metrics that correlate best with the subjective preference are those based on the image plane distributions (i.e., the Strehl ratio, the entropy, ...) rather than others based on the wavefront aberration error measured on the pupil plane.

From the calculation of the output pattern we have used a metric based on the variation of the Strehl ratio with the propagated distance. This parameter is obtained as the as the quotient between the peak of the point spread function (PSF) provided by an aberrated system at a desired plane and the peak of the PSF of a diffraction-limited system. Since we are using input beams coming from the infinite, the PSF is just the intensity distribution obtained with the above described method.

\section{CHROMATIC DIFFERENCE OF REFRACTION}

The chromatic difference of refraction is given ${ }^{30}$ by

$$
R_{x}(\lambda)=\frac{\left[n_{v}(\lambda)-n_{v}(\bar{\lambda})\right] F(\bar{\lambda})}{n_{v}(\bar{\lambda})}-\Delta F(\lambda)
$$

with

$$
F(\bar{\lambda})=\frac{n_{v}}{z_{m}(\bar{\lambda})}
$$

where $z_{m}(\bar{\lambda})$ is the plane of best image for the reference wavelength $\bar{\lambda}=590 \mathrm{~nm}$ and $\Delta F(\dot{\lambda})=F(\lambda)-F(\bar{\lambda})$ is the chromatic difference of power.

Atchison and Smith ${ }^{31}$ show that equation (8) can be approximately expressed by

$$
R_{x}(\lambda)=-\frac{\left[n_{v}(\lambda)-n_{v}(\bar{\lambda})\right] F(\bar{\lambda})}{n_{v}(\bar{\lambda})\left[n_{v}(\bar{\lambda})-1\right]}
$$

In that reference suppose that the chromatic difference in refractive error $R_{x}(\lambda)$, at least for a reduced eye, will have the same form as the dispersion equation for the refractive index. Therefore, if the refractive index is well fitted by Cauchy's equation, so will be the chromatic difference of refraction.

$$
R_{x}(\lambda)=1.60911-6.70941 \times 10^{+5} / \lambda^{2}+5.55334 \times 10^{+10} / \lambda^{4}-5.59998 \times 10^{+15} / \lambda^{6}
$$


Thibos et al. ${ }^{6}$ used the Cornu equation, with their data to obtain

$$
R_{x}(\lambda)=1.68524-633.46 /(\lambda-214.102)
$$

Both adjustments will be compared with our own results.

\section{RESULTS}

With the diffractive method we have analyzed longitudinal chromatic aberration in ten young emmetropic eyes. In Figure 2. we have represented an example of the variation of the Strehl ratio with the propagation distance in the posterior chamber. The maximum of the distribution indicates the best image plane. We have represented the situation for three different wavelengths $(\lambda=450 \mathrm{~nm}, \lambda=550 \mathrm{~nm}$ and $\lambda=650 \mathrm{~nm})$ with a natural pupil of $4 \mathrm{~mm}$ of diameter. Notice that the effect of the chromatic aberration consists of a displacement of the whole curve, but the evolution of the curve with $z$ is not altered very much. This means that changes in wavelength have little effect on other aberrations except for defocusing. If we consider that the retinal plane is located at $z=24.1 \mathrm{~mm}$, notice the Strehl ratio for other wavelengths but $\lambda=550 \mathrm{~nm}$ decreases dramatically. Moreover, the effect of these wavelengths will be also attenuated by the spectral sensitivity curve of the retina, so that its contribution to the total quality of the image is very low.

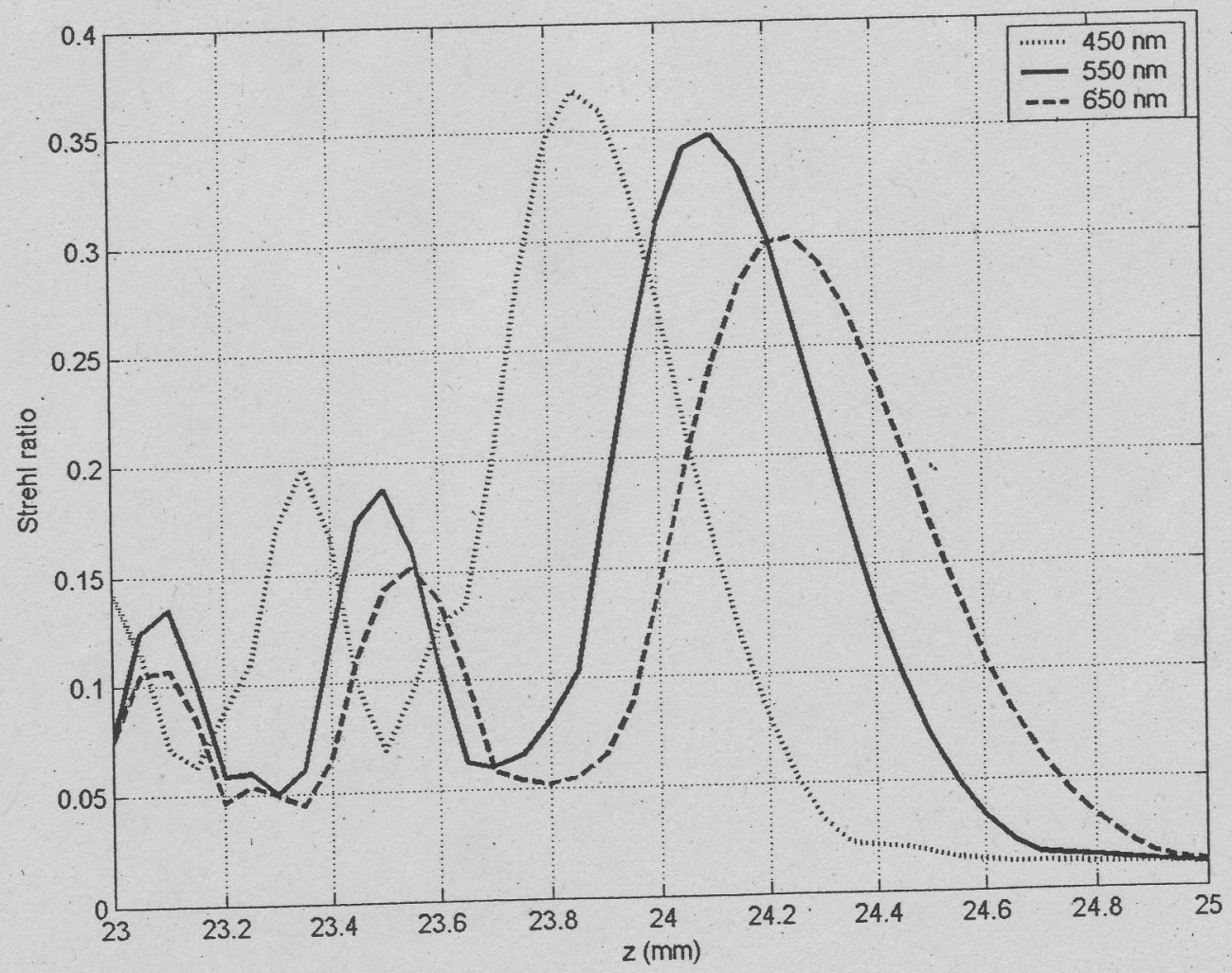

Figure 2: Strehl ratio variation with propagated distance $z$ for three different wavelengths in a normal eye for a pupil diameter of $4 \mathrm{~mm}$.

We have also used the described method for the LCA calculation of 10 eyes in the visible spectrum and around infrared wavelengths. A four-term Cauchy equation has been fitted to the mean of all the eyes resulting: 


$$
R_{x}(\lambda)=1.6905-7.6009 \times 10^{+5} / \lambda^{2}+7.9524 \times 10^{+10} / \lambda^{4}-6.8854 \times 10^{+15} / \lambda^{6}
$$

In Figure 3 we present the mean of the chromatic difference of refraction in the visible spectrum range of the ten subjects obtained with the diffractive method compared with equations (11) and (12). We can observe there that our results are in agreement with those results.

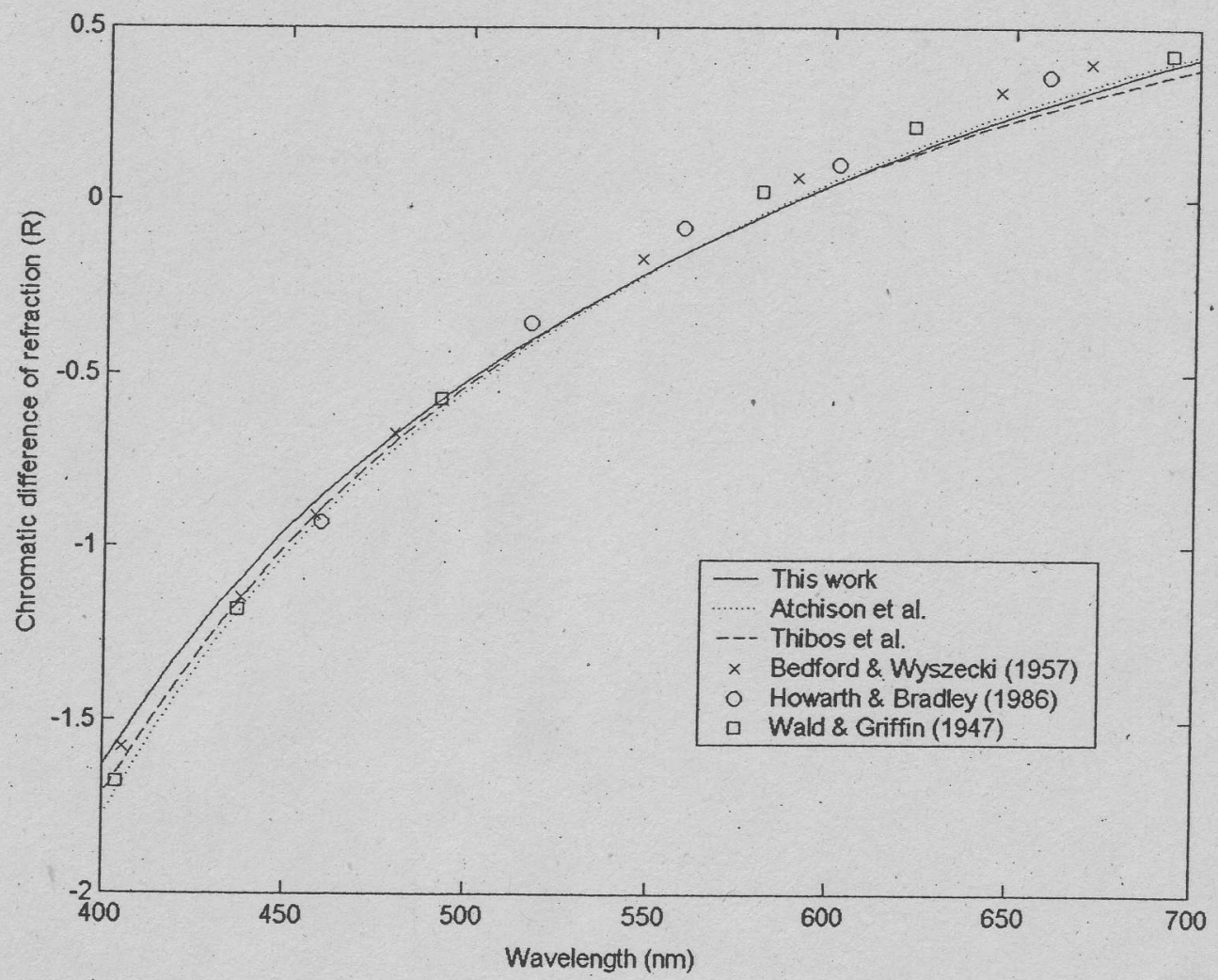

Figure 3. Chromatic difference of refraction from three experimental studies in the visible spectrum, solid line represent the mean chromatic difference of refraction of ten subjects, best-fit Cornu's equation used by Thibos et al. ${ }^{6}$ and Cauchy equation used by Atchison et al. ${ }^{31}$. All data were set to be zero at $590 \mathrm{~nm}$.

Figure 4 presents the average defocus from the ten subjects as a function of wavelength. Before averaging, data were shifted along the vertical axis to cancel the defocus at the shortest wavelength $(700 \mathrm{~nm})^{12}$. The changes of chromatic difference of refraction with wavelength in the near infrared range were accurately fitted by a linear equation. The red line represents this linear fit, whose equation is:

$$
\begin{aligned}
& R(\lambda)=0.0022(\lambda-700)-1.5345 \\
& r^{2}=0.9930
\end{aligned}
$$

This result is in good agreement with the linear fit performed in bibliography ${ }^{12}$

$$
\begin{aligned}
& R(\lambda)=0.0021(\lambda-700)-1.4341 \\
& r^{2}=0.9937
\end{aligned}
$$


With this method, we have characterized the dependence of aberrations with wavelength in the visible and near infrared spectrum range showing that our results are in good agreement with others from bibliography and with the advantage of using an objective method.

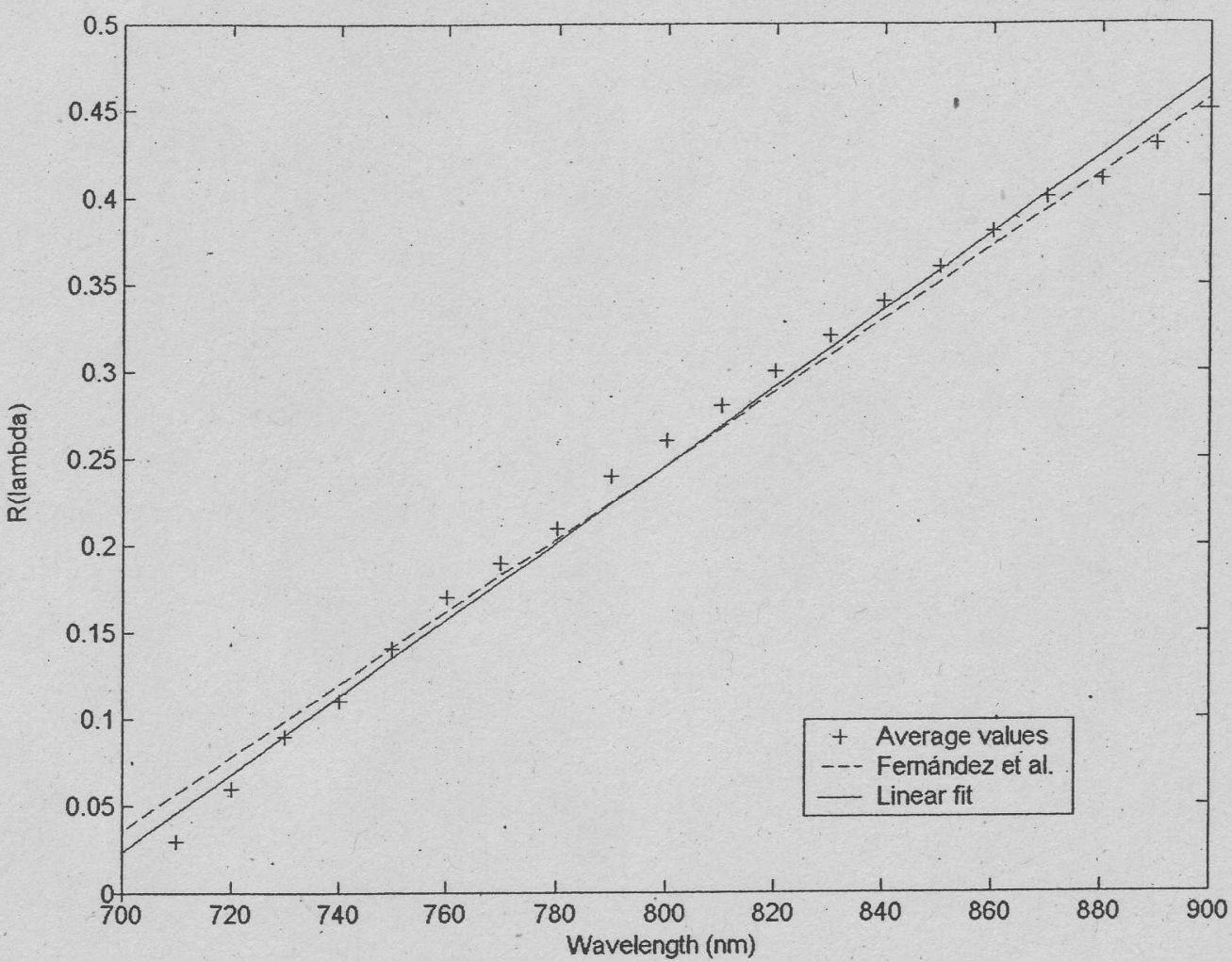

Figure 4 Chromatic difference of refraction from the mean of the chromatic difference of refraction computed from ten subjects in the nearby infrared range, linear fit and linear fit of Fernández et al ${ }^{12}$.

As an application of the method we have analyzed the chromatic aberrations in a highly aberrated eye, i.e. a keratoconus. This malformation produces irregular astigmatism and other high order aberrations. Hence, quality of vision is poor. In figure 5, we show the evolution of the Strehl ratio with the propagated distance $z$, for two different wavelengths and for a pupil size of $4 \mathrm{~mm}$ of diameter. The values of the Strehl ratio are extremely low, and no good-vision peak can be detected. Notice that now the curves change with the wavelength, which means that this parameter affects to the rest of aberrations. The wavelength affects to the index of refraction and this one to the focal length of the element. Changing the focal length in such eye may introduce decoupled variations in all aberration terms. In figure 5 we also reproduce the case of the normal eye for comparison purposes. 


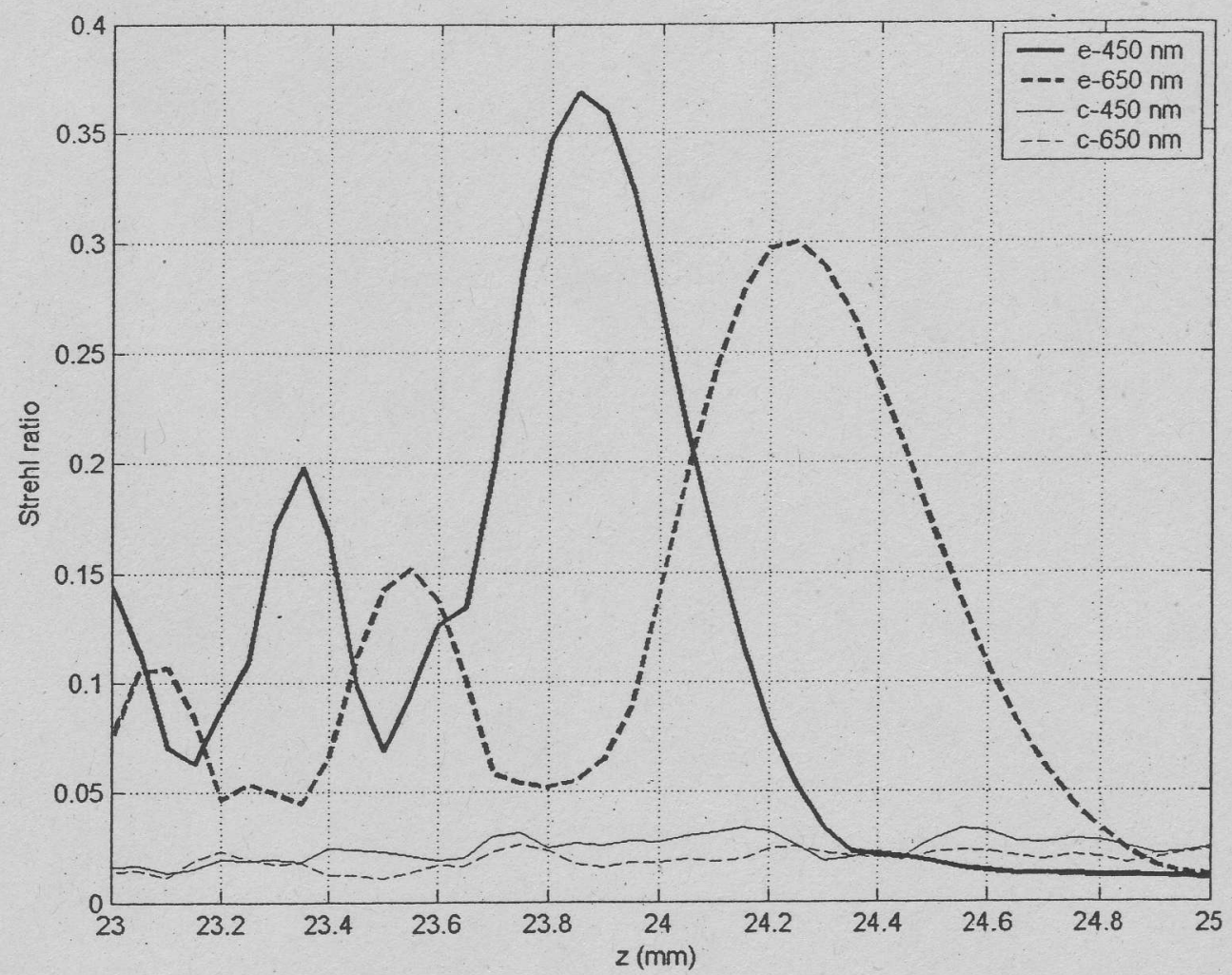

Figure 5: Strehl ratio variation with propagated distance $z$ for two different wavelengths. With $e$ - we denote the curves for an emmetropic eye. $c$ - refers to an eye affected of keratoconus. Both eyes are calculated for pupil diameters of $4 \mathrm{~mm}$.

\section{REFERENCES}

1. Wald, G. and Griffin, D. T., "The change in refractive power of the human eye in dim and bright light", J. Opt. Soc. Am. 37 (321-329), 1947.

2. Bedford, R. E. and Wyszecki, G., "Axial chromatic aberration of the human eye", J. Opt. Soc. Am. 47 (564565), 1957.

3. Charman W. N. and Jennings, J. A. M. "Objective measurements of the longitudinal chromatic aberration of the human eye”. Vision Res., 16 (999-1005), 1976.

4. Howarth, P. A. and Bradley, A., "The longitudinal chromatic aberration across the retina of the human eye", Vision Res. 16 (361-366), 1986.

5. Thibos, L. N., Bradley, A., Still, D. L., Zhang, X. and Howarth, P. A., "Theory and measurement of ocular chromatic aberration", Vision Res. 30 (33-49), 1990.

6. Thibos, L. N., Ye, M., Zhang, X. and Bradley, A., "The chromatic eye: a new reduced-eye model of ocular chromatic aberration in humans", Appl. Opt. 31 (3594-3600), 1992.

7. Rynders, M. C., Navarro, R. and Losada, M. A., "Objective measurement of the off-axis longitudinal chromatic aberration in the human eye". Vision Res. 38(513-522), 1994.

8. Marcos, S., Burns, S. A., Moreno-Barriuso, E., Navarro, R., "A new approach to the study of ocular chromatic aberrations", Vision Res. 39 (4309-4323), 1999. 
9. McLellan, J. S. Marcos S., Prieto P. M. and Bums, S. A. "Imperfect optics may be the eye's defense against chromatic blur", Nature 417 (174-176), 2002.

10. Thibos, L. N., "Calculation of the influence of lateral chromatic aberration on image quality across the visual field", J. Opt. Soc. Am. A, 8 (1673-1680), 1987.

11. Thibos, L. N., Bradley, A and Zhang, X., "The effect of ocular chromatic aberration on monocular visual performance", Optom. Vis. Sci. 68, 599-607 (1991)

12. Fernández, E. J., Unterhuber, A., Prieto, P. M., Hermann, B., Drexler, W. and Artal, P., "Ocular aberrations as a function of wavelength in the near infrared measured with a femtosecond laser", Opt. Express 13 (400-409), 2005.

13. Mas, D., García, J., Ferreira, C., Bernardo, L. M. and Marinho, F. J., "Fast algorithms for free-space diffraction pattern calculation", Opt. Comun., 164 (233-245), 1999.

14. Mas, D., Pérez, J., Hernández, C., Vázquez, C., Miret, J. J. and Illueca, C., "Fast numerical calculation of Fresnel patterns in convergent systems", Opt. Comun, 227 (245-258), 2003.

15. Pons, A. M., Lorente, A., Illueca, C., Mas, D. and Artigas, J. M., "Fresnel diffraction in a theoretical eye: a fractional Fourier transform approach", J. Mod. Opt., 46 (1043-1050), 1999.

16. Illueca, C., Mas, D., Pérez, J., Pons, A. M. and Artigas J. M., "Refractive analysis of the human cornea through propagated fields", J. Mod. Opt., 48 (811-829), 2001.

17. Mas, D., Pérez, J., Vázquez, C., Hernández, C. and Illueca, C., "Near-field light distributions propagated from human corneas: determination of relevant patterns", J. Mod. Opt., 50 (1335-1352), 2003.

18. Pérez, J., Mas, D., Miret, J.J., Vázquez, C., Hernández, C. and Illueca, C., "Fresnel based analysis of Kasprzak's crystalline model: statistical results and individual predictions", Optik, 116 (49-57), 2005.

19. Pérez, J.; Mas, D., Illueca, C., Miret, J.J., Vázquez, C. and Hernández, C., "Complete algorithm for the calculation light patterns inside the ocular media", J. Modern Optics, 52 (1161-1176), 2005.

20. Cauchy, A. L., Mémoire sur la dispersion de la lumière, Nouveaux Exercises de Mathématiques, in Oeuvres Complètes d'Agustin Cauchy, 2nd Series, Vol. 10, Gauthier-Villars et Fils, Paris, 1895.

21. Dubbelman, M. and Van der Heijde, G. L., "The shape of the aging human lens: curvature, equivalent refractive index and the lens paradox", Vision Res. 41 (1867-1877), 2001.

22. Siedlecki, D., Kasprzak, H., and Pierscionek, B.K., "Schematic eye with a gradient-index lens and aspheric surfaces", Opt. Lett. 29 (1197-1199), 2004.

23. Emsley, H. H., Visual Optics, Vol. 1, Butterworth, London, 1924.

24. Gullstrand, A., "Apendix II. Optical imagery", in H. von Helmtholtz, Handbuch der Physiologischen Optik, 3rd ed. Leopold Voss, Hamburg, 1909. English translation, Helmholtz's Treatise on Physiological Optics, J. P. C. Southall, ed., O.S.A., Washington, D. C., Vol. 1, 1924.

25. Le Grand, Y. and Hage, S. G., Physiological Optics, Springer-Verlag, Berlin, 1981.

26. Kooijman, A. C., "Light distribution on the retina of a wide-angle theoretical eye", J. Opt. Soc. Am. 73 (15441550), 1983.

27. Guirao, A. and Williams, D. R., "A method to predict refractive errors from wave aberration data", Optom. Vis. Sci. 80 (36-42), 2003.

28. Marsack, J. D., Thibos, L. N., and Applegate, R., "Metrics of optical quality derived from wave aberrations predict visual performance", J. Vision, 4 (322-328), 2004.

29. Thibos, L., Hong, X., Bradley, A. and Applegate, R., "Accuracy and precision for objective refraction form wavefront aberrations", J. Vision 4 (329-351), 2004.

30. Atchison, D. A. and Smith, G., Optics of the Human Eye, Butterworth-Heinnemann, Oxford, UK, 2000.

31. Atchison, D. A. and Smith, G., "Chromatic dispersions of the ocular media of human eyes". J. Opt. Soc. Am. 22 (22-37), 2005. 


\section{ACKNOWLEDGMENTS}

This work has been partially supported by the Conselleria de Cultura, Educació i Esport of the Generalitat Valenciana, through the project nr. GV04A/578. 